\section{The usefulness of} the Retinomax autorefractor for childhood screening validated against a Danish preterm cohort examined at the age of 4 years
HC Fledelius, R Bangsgaard, C Slidsborg and $\mathrm{M}$ laCour
Copenhagen University Eye Department, Glostrup Hospital \& Rigshospitalet, Copenhagen, Denmark

Correspondence: HC Fledelius, Copenhagen University Eye Department, Glostrup Hospital \& Rigshospitalet, Eye Clinic E 2061, Rigshospitalet, Blegdamsvej 9, 2100 Copenhagen, Denmark Tel: +45 3545 2064; Fax: +45 35452098. E-mail: hcfled@mail.dk

Received: 22 September 2014

Accepted in revised form:

7 January 2015

Published online:

20 March 2015
Abstract

Background and purpose Refractometers have gained a foothold in childhood screening for ophthalmic disorders. Given the results of an ophthalmic follow-up of an extremely preterm Danish cohort, the results of the Retinomax autorefractor were further evaluated.

Materials and methods A nationwide cohort of infants born before gestational age 28 weeks $(n=178)$ and 56 term controls were examined at the age of 4 years. Refraction was given as the cycloplegic Retinomax value. For this study, we analysed the equipment's confidence value on the printout and equipment-induced myopization (as the difference between refraction measured before and after topical cyclopentholate $1 \%$ ), both items hypothetical with a view to having identified factual ophthalmic deviations. Results Thirty-two of $\mathbf{4 2}$ eyes with visual acuity $\leq \mathbf{0 . 4}$ had high Retinomax confidence values (8-9); the Retinomax values were also high in 10 of 12 children with strabismus and lack of stereopsis. Low values (1-6) were recorded in 11 single eyes, 5 of which were normal (false positives). Three children already known to have low vision were unable to cooperate. The overall mean value for equipment-induced myopization was 1.9 D (range, 0-6.87 D). Myopization showed no correlation with visual acuity and corneal curvature, and a weak positive correlation with refractive value disappeared when the myopic outliers were excluded. Conclusions The hand-held Retinomax seemed to be reliable for assessing refraction in 4-year-old children, provided a cycloplegic agent is applied; if used alone, the Retinomax would have missed several cases of ophthalmic deviation during screening. Equipment-induced myopization was not indicative.

Eye (2015) 29, 742-747; doi:10.1038/eye.2015.14; published online 20 March 2015

Introduction

Early identification of paediatric ophthalmic problems is of obvious importance for visual care in childhood. This applies to the detection not only of serious eye pathology but also functional loss due to amblyopia. Both aspects have been targeted in Scandinavia for decades at annual childhood examinations by family doctors $^{1}$ or in child health care centres..$^{2-4}$ Subsequent paediatric ophthalmic measures have radically reduced deep amblyopia in preschool children. ${ }^{3,5}$

Preschool visual acuity screening can generally be conducted with high testability and good detection of anomalies from age 3-4 years. Trained health personnel are required, ideally with backup from a full team headed by paediatric ophthalmologists. From a worldwide perspective, limits are set by the socioeconomic infrastructure, ${ }^{6}$ and simpler settings are required..$^{7-9}$

The new generations of autorefractometers led to high expectations because they can be serviced by less specialized personnel. High testability in non-cycloplegic cohorts was thus reported in several centres using the hand-held Retinomax autorefractometer (Nikon Inc, Tokyo, Japan), and positive findings were subsequently evaluated against cycloplegic paediatric 
ophthalmic examinations. ${ }^{10-18}$ Between the ages of 2 and 4 years, the testability of single devices increased from about 50 to $90 \%$; however, some conditions remain undetected. The Retinomax can identify significant ametropia immediately but poor cooperation from the child should further direct attention to deviations from normal. For example, there could be reduced confidence values due to off-axis fixation in strabismus, as well as in brain disorders.

A recent Danish paediatric ophthalmic cohort study on the effects of extreme prematurity in survivors now aged 3-4 years was conducted with a primary focus on development (retina or brain) when visual ability was subnormal. ${ }^{19}$ The refractive value was taken from the cycloplegic Retinomax printout after a single application of cyclopentholate $1 \%$ eye drops. An initial recording taken before insertion of the eye drops was used to estimate equipment-induced myopization (EIM). In addition to the Retinomax confidence value, this functional parameter allowed us to test the overall suitability of the Retinomax for screening in the field.

The set-up of the basic follow-up study permitted several questions to be addressed. (1) In a clinical sample, how many children could be evaluated with the Retinomax? (2) How did the equipment-generated confidence value (a technical score, ideally at least 8 , on a scale up to 10) relate to the issue of false positives (a low confidence value despite normal function) and false negatives (a high confidence value and ophthalmic disorder/dysfunction)? (3) For screening purposes, as hypothesised in the present analysis, can non-cycloplegic recordings alone be useful? The proximity of the refractometer to the eye can often trigger accommodation (EIM) and, although virtually a confounder this could provide factual guidance if a systematic trend is apparent over the refractive profile. (4) Amblyopia (given by an individual difference between the eyes in best-corrected visual acuity (BCVA) of at least two lines on the logMAR chart) was briefly addressed.

\section{Materials and methods}

Survivors from a national preterm birth cohort born between February 2004 and March 2006 at a gestational age of $<28$ weeks were asked to attend for eye examination around corrected age of 4 years. ${ }^{19-21}$ Eventually 178 preterm and 56 full-term controls were able to participate.

All preterm children had early data on retinopathy of prematurity (ROP) on record. No ROP had been recorded in 105 children, reversible ROP (stages 1-3) was found in 41 and 32 had diode laser treatment for threshold ROP. ${ }^{19-21}$
Refractive data were assessed with a hand-held Retinomax K-plus3 auto-keratorefractometer (Nikon Inc) before application of cyclopentholate $1 \%$ eye drops and 30-50 min after instillation. The equipment was used in normal mode, with automated fogging of the fixation target to minimise accommodation. All children had several trials for optimum results; four preterm and three full-term children could not cooperate satisfactorily. Two items were emphasised in the present study: the alignment and optical clarity mark of the Retinomax procedure (the confidence value, on a scale from 1 to 10) and the EIM. A confidence value of at least 8 generally signifies good technical quality of measurement. ${ }^{10-13}$ Overall, the spherical equivalent refraction (SER), calculated as sphere $+\frac{1}{2}$ cylinder (in dioptres), could be given for 174 of 178 preterm children and 53 of 56 fullterm controls. The difference between pre-cycloplegic and cycloplegic values expressed the EIM. Keratometry results were missing on the printouts for 23 preterm and 5 full-term children.

Best-corrected single eye and binocular visual acuity was tested on a HOTV logMAR chart at a distance of $3 \mathrm{~m}$. This was feasible in 227 of the 234 children. Teller card acuity was obtained for seven children. In the text, Snellen equivalents are presented as decimal values.

Binocular status was assessed by testing eye motility, the Hirschberg test (corneal pencil light reflex for symmetric central fixation) and the cover test. The Lang II test was used to assess stereopsis.7,19

The GraphPad4 Prism programme (GraphPad Software Inc., La Jolla, CA, USA) was used to handle the data and evaluate the statistics (non-parametric, Mann-Whitney; parametric $t$-test, correlation and regression; $P$-level $<0.05)$.

The study was conducted in accordance with the Helsinki Declaration and was approved by the Ethical Committee of Copenhagen and the Danish Data Protection Agency.

\section{Results}

The Retinomax recording under cycloplegia was accepted as the refractive value for each individual. The results are discussed in a separate publication, along with a literature review of refraction in preterm children. ${ }^{21}$ The Retinomax examination was performed in $95.3 \%$ of the 234 participants.

A high Retinomax confidence value (for technical alignment and free access to the infrared target beam) was the hallmark of the series. Values of 8 and 9 were achieved in most cases, and there was no statistically significant difference between preterm and full-term children. The 32 laser-treated stage 3 ROP cases only marginally sorted out; 29 had bilateral confidence values 
$\geq 7$, the median value was 8 and 3 children could not be tested. Comparing the confidence values with other data from the study, some findings deserve mention.

Low confidence values (from 1 to 6 ) were found in 11 eyes in the pooled series, as a unilateral score in all cases. The BCVA was 0.8-1.0 in 5 eyes, 0.5-0.6 in 3 eyes, and low vision was found in 3 eyes (BCVA 0.25-0.1). An error was indicated in 3 individuals with reduced vision (logMAR BCVA 0.2/0.4 and 0.3/0.32 in 2 children, and a Teller estimate of 0.1 in the better eye of the 3rd child who was aphakic and had severe brain damage). Using a low or missing confidence number as the sole criterion, only 14 of the 234 children aged 4 years (6\%) would have been identified as candidates for full paediatric ophthalmic status on the suspicion of reduced visual acuity, and 5 would have been false positives.

Forty-two eyes had visual acuity $\leq 0.4$. In this subgroup, with the obvious possibility of amblyopia or other conditions in need of therapy, 32 eyes presented confidence values of 8 or 9 and would appear as false negatives during a screening based on Retinomax. Confidence values of 7 and 6 were found in 6 and 2 eyes, respectively. A preterm child with BCVA 0.4/0.11 had confidence values of 8 and 1, which indicates a false negative in the right eye and correctly positive in the left eye.
The Retinomax recordings for 21 preterm children presented in Table 1 represent a selection of children in whom the customary screening aims (such as cycloplegic refractive error, heterotropia and/or reduced visual acuity) would not have been correctly identified by the confidence values. Heterotropia was recorded in 15 children (10 eso, 4 exo, and 1 hyper), giving an incidence of $8.4 \%$ (vs none in the controls), and 12 of the 15 children had no stereopsis. Stereopsis was not found in another 13 preterm children (not shown in the table), although no motility disorder was identified, and they also had normal confidence values. Among the controls, there was one child without stereopsis; he had normal visual acuity and his Retinomax recording was not available.

As mentioned briefly, amblyopia (an individual BCVA side difference of at least two lines) presented no trend for Retinomax testability with regard to better/worse eye.

EIM is the involuntary accommodative response triggered in the measuring situation by any instrument, here the Retinomax, when unintentionally conceived as near. ${ }^{22-25}$ The cycloplegic refractive value was subtracted from the initial native value and presented as the SER. Instrument myopia was similar in the two main groups in the study, preterm and full-term (Figure 1). The values calculated for both groups ranged from $0 \mathrm{D}$ to almost $7 \mathrm{D}$, and the mean values were similar $1.90 \mathrm{D}(1.11)$ and $1.91 \mathrm{D}$

Table 1 Retinomax confidence values (see text) compared with customary childhood screening targets such as ametropia, strabismus, anisometropia and amblyopia/reduced vision, among 21 of the extremely preterm children (gestational age $<28$ weeks; $n=178$ ) now aged 4 years

\begin{tabular}{|c|c|c|c|c|c|c|c|}
\hline \multirow{2}{*}{$\begin{array}{l}\text { Original serial } \\
\text { case number }\end{array}$} & \multicolumn{2}{|c|}{ Retinomax confidence value } & \multirow[t]{2}{*}{ Strabismus } & \multicolumn{2}{|c|}{ Refraction (SER, in D) } & \multicolumn{2}{|c|}{ Best-corrected visual acuity } \\
\hline & Right eye & Left eye & & Right eye & Left eye & Right eye & Left eye \\
\hline 24 & 7 & 4 & ET & -1.62 & 0.3 & 0.8 & 0.8 \\
\hline 234 & 8 & 1 & ET & 3.12 & 3.25 & 0.4 & 0.5 \\
\hline 219 & 7 & 7 & - & -4.12 & -4.25 & 0.4 & 0.4 \\
\hline 235 & 8 & 1 & - & 1.5 & 1.37 & 1.0 & 1.0 \\
\hline 110 & 8 & 2 & - & 0.12 & -9.12 & 0.4 & 0.11 \\
\hline 59 & 6 & 7 & - & 2.12 & 2.37 & 0.2 & 0.05 \\
\hline 99 & 7 & 6 & - & 2.0 & 2.0 & 0.4 & 0.25 \\
\hline 16 & 9 & 9 & - & -2.25 & 1.0 & 0.3 & 0.8 \\
\hline 2 & 8 & 8 & ET & 0.87 & -2.25 & 0.4 & 0.4 \\
\hline 6 & 8 & 8 & ET & -0.5 & -4.37 & 0.63 & 0.25 \\
\hline 7 & 8 & 8 & $\mathrm{XT}$ & 1.0 & 1.37 & 0.8 & 0.5 \\
\hline 55 & NA & NA & XT & NA & NA & 0.16 & 0.4 \\
\hline 126 & 8 & 8 & ET & -0.25 & -0.12 & 0.4 & 0.4 \\
\hline 129 & 9 & 10 & ET & 3.12 & 3.0 & 0.4 & 0.63 \\
\hline 183 & NA & NA & ET & 2.25 & 2.37 & 032 & 0.32 \\
\hline 214 & 9 & 10 & $\mathrm{XT}$ & 0.87 & 0.87 & 0.4 & 0.63 \\
\hline 227 & 8 & 8 & $\mathrm{XT}$ & 1.12 & 1.62 & 0.4 & 0.4 \\
\hline 253 & 9 & 9 & ET & 1.25 & 0.87 & 0.24 & 0.24 \\
\hline 4 & 9 & 8 & ET & 1.25 & 1.25 & 0.8 & 0.49 \\
\hline 109 & 8 & 8 & ITX & 1.25 & 1.25 & 0.8 & 0.8 \\
\hline 98 & 9 & 9 & HT & 1.25 & 1.25 & 0.8 & 0.8 \\
\hline
\end{tabular}

Abbreviations: ET, esotropia; HT, hypertropia; IXT, intermittent exotropia; NA, data not available; SER, spherical equivalent refraction; XT, exotropia. The seven children with subnormal confidence scores $(\leq 7)$ are shown at the top. 


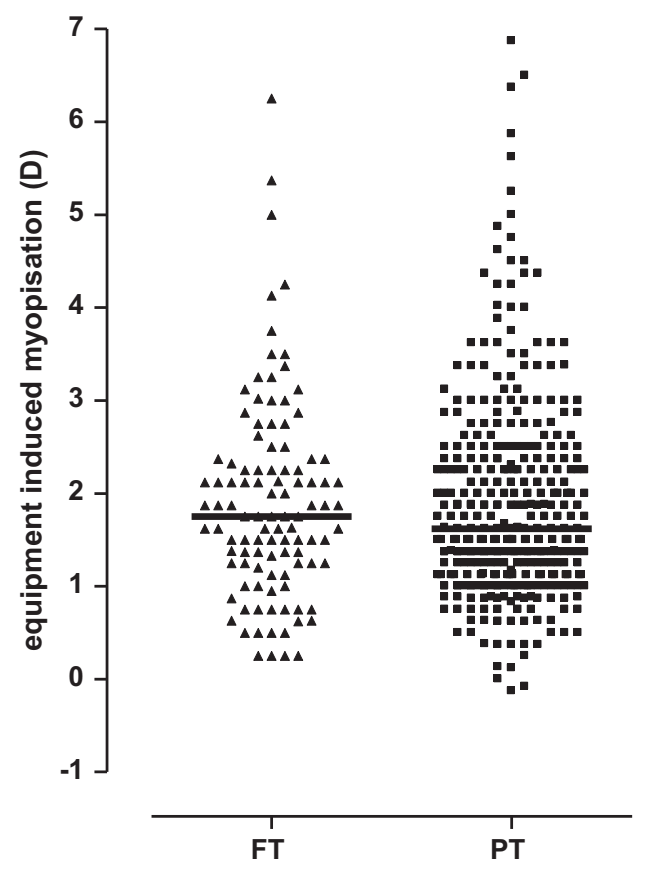

Figure 1 Equipment-induced myopization (Retinomax) in preterm children (squares) and full-term controls (triangles), calculated as the individual Retinomax SER value after the application of cyclopentholate subtracted from the recording before the eye drops. Median values were $1.75 \mathrm{D}$ and $1.62 \mathrm{D}$, respectively (Mann-Whitney $P=0.66$ ).

(1.08); median values were $1.62 \mathrm{D}$ and $1.75 \mathrm{D}$, respectively; $P>0.05)$. No statistically significant differences were demonstrated according to the presence and the degree of ROP (Figure 2).

We then compared the EIM estimates with the other main parameters under study, namely refraction, corneal curvature radius, and visual acuity. From the SER profiles (Figures 1 and 2), it was obvious that a central most normal core was shared by the preterm children and the controls. We therefore focused on the 2 marginal subgroups that characterized the preterm group: (a) those who had myopia (a total of 16 eyes), and (b) those with high hyperopes who had not orderly emmetropised (45 eyes) (Table 2). The small group of myopic eyes had a lower EIM value, the high hyperopes had a higher value, and the slope of the regression line $(P<0.0001)$ was positive due mainly to these relatively few outliers. The trends were identical in both eyes. The regression line for the right eye was expressed by $y=1.43+0.356 x, r=0.33$ (Figure 3). However, the significance of the positive slope was neutralised if the few myopic outliers were excluded from the calculations.

No correlation was established when the EIM values were compared with the radius of the corneal curvature $(r=0.064, P=0.36)$ and BCVA $(r=0.047, P=0.48)$. In addition, the 40 eye pairs with amblyopia were not differentiated.

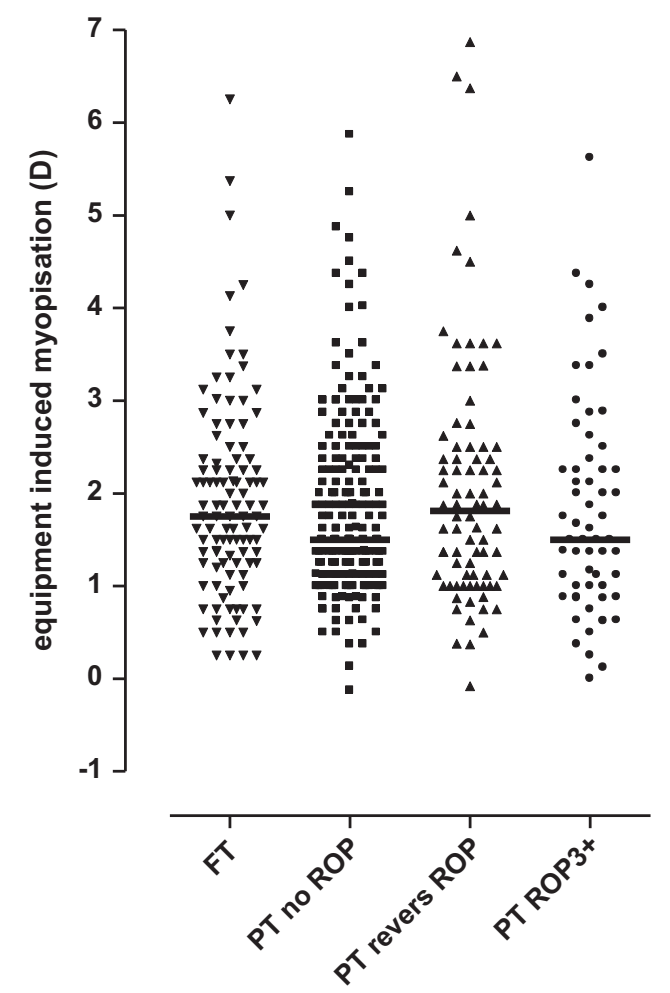

Figure 2 Equipment-induced myopization (Retinomax) in the series, with preterm children subdivided by the appearance and stage of retinopathy of prematurity. Dunn's multiple comparison tests: not significant. Full-terms = triangles base up. Squares, triangles base down, and dots specify the preterm subgroups.

Table 2 Equipment-induced myopization in the preterm group, with the two marginal SER refractive groups specified: hyperopia $\geq+2.5 \mathrm{D}$ and negative refraction (myopia)

\begin{tabular}{lccc}
\hline Group & \multicolumn{3}{c}{ Equipment-induced myopization (D) } \\
\cline { 2 - 4 } & Mean value (SD) & Median value & Range \\
\hline PT myopes (16 eyes) & $1.22(0.95)$ & 1.15 & $0-3.88$ \\
PT central (285 eyes) & $1.83(1.05)$ & 1.5 & $0-6.89$ \\
PT hyperopes (47 eyes) & $2.54(1.25)$ & 2.5 & $0-6.37$ \\
\hline
\end{tabular}

Abbreviations: PT, preterm; SD, standard deviation; SER, spherical equivalent refraction.

\section{Discussion}

All Retinomax recordings were performed by the same senior paediatric ophthalmologist (RB). As background for the present analyses, an impression over years of daily use of the equipment had suggested an association between poor recordings and ophthalmic problems. Compared with the findings from the basic study, the Retinomax printouts were evaluated from a simulated screening perspective, focusing on the Retinomax confidence value and EIM. 


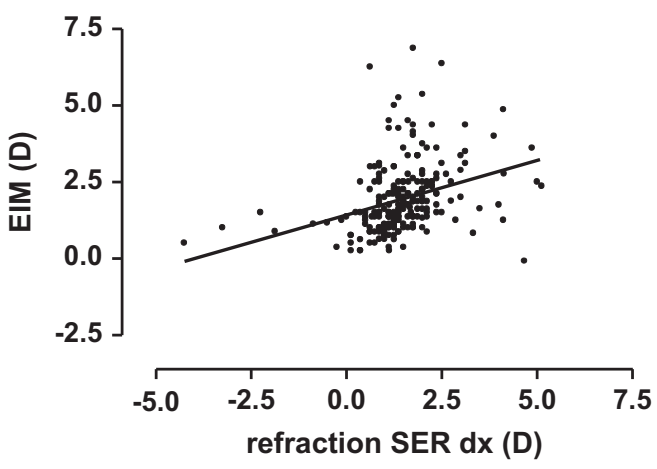

Figure 3 A weak correlation $(r=0.33)$ was found between equipment-induced myopia by Retinomax and the cycloplegic refractive level, based on all right eyes in the full sample. The regression line is given by $y=1.43+0.356 x(r=0.33)$; however, the significance of the slope disappeared when the few myopic outliers were omitted from the calculations.

From the perspective of ophthalmic screening for refractive level, strabismus, anisometropia, and subnormal visual acuity, a total of 21 children were included in Table 1. For the seven children listed at the top, subnormal Retinomax confidence values correctly indicated states that should be spotted during screening. At the bottom, 12 children with high Retinomax scores present as failures (false negatives), and 2 were recorded as not available. Overall, this means that a large proportion of children with ophthalmic parameters in need of detection might have remained undetected if the Retinomax confidence value was used as primary screening criterion. From the data not included in the table, however, the Lang II stereopsis test would have spotted most of these cases.

A systematic trend suggested lower EIM values in the myopic tail, and higher values among the high hyperopes (Table 1; Figure 3). At all refractive levels, however, the variation seemed unpredictable, and empirical correction factors based on non-cycloplegic refractometer printouts could not be outlined. Specific EIM responses were not found to be useful for detecting subnormal vision/amblyopia.

A limitation of the study may pertain to the repeatability of the autorefractor measurements, even when under cycloplegia. In recent studies, such recordings have replaced time-consuming and operatordependent subjective trials with glasses (BCVA) after conventional retinoscopy. For the present study, the latter approach would have resulted in many dropouts, considering the attention span of the 4-year-olds and the programme for the day. Individual autorefractor results may, however, show variation, and the possibility of instrument-based outliers should be considered. ${ }^{26}$

Another possible limitation of the study is the degree of cycloplegia. To maintain the goodwill of the children, our choice was cyclopentholate $1 \%$ given just once. However, as the iris in most native Danes is light in colour, ${ }^{27}$ the method was trusted; usually children cannot see close up for hours after just one drop. Nevertheless, we cannot exclude having missed some high hyperopic dioptres, in particular among those who had not emmetropized. The myopes, conversely, had low scores for equipment-induced myopic shift. This might be a consequence of reduced need for accommodation in myopes combined with their acquaintance with the refractometer and vision testing on previous occasions. For the evaluation of EIM, the onedrop-only regimen provides a conservative estimate.

We further acknowledge that our test sample of 4-yearold extremely preterm survivors was specially selected. However, the higher expectancy of abnormal eye findings is regarded as a strength of the study, to which can be added the level of expert paediatric ophthalmology in the basic study.

In conclusion, the main findings in our series were as follows: (a) there was a factual indication under cycloplegia of significant refractive error, (b) there was wide and unsystematic variation in EIM in children who were not cycloplegic, and (c) children who refuse or are unable to cooperate with the Retinomax procedure should be given attention.

In general, a poor confidence value with the Retinomax was a rare finding in the series. It was associated with reduced vision in less than half such cases, and in a screening context they would have been labelled positive. On the contrary, most of the eyes with reduced vision would have passed the test as false negatives, and even confidence value scores of 9 and 10 occurred. The results support the customary use of more than just one tool for early childhood ophthalmic screening in the field. Clearly, simple binocular vision tests are useful in combination, and should be used in particular when strabismus and ametropia are being assessed in early childhood screening.

Summary

What was known before

- The testability and effectiveness of modern autorefractometers, particularly the Retinomax equipment, have been emphasised in large-scale ophthalmic preschool screening studies. The potential for spotting ophthalmic deviations even seems to go beyond the basic demonstration of ametropia.

What this study adds

- Weighted against paediatric ophthalmic findings in an extremely preterm Danish cohort aged 4 years, the high testability of the Retinomax autorefractometer is confirmed. Analysis of the equipment's confidence values, however, gave many false-positive and false-negative results. We also analysed equipment-induced (relative) myopization. No systematic trends were revealed. In particular, empirical correction factors for non-cycloplegic results could not be given. 


\section{Conflict of interest}

The authors declare no conflict of interest.

\section{References}

1 Jensen H, Goldschmidt E. Visual acuity in Danish school children. Acta Ophthalmol (Copenh) 1986; 64: 187-191.

2 Köhler L, Stigmar G. Visual disorders in 7-year-old children with and without previous visual screening. Acta Paediatr Scand 1978; 67: 373-377.

3 Kvarnström G, Jakobsson P, Lennerstrand G. Visual screening of Swedish children: an ophthalmological evaluation. Acta Ophthalmol Scand 2001; 79: 240-244.

4 Ohlsson J, Villareal G, Sjöström A, Abrahamsson M, Sjöstrand J. Visual acuity, residual amblyopia and ocular pathology in a screened population of 12-13 year-old children in Sweden. Acta Ophthalmol Scand 2001; 79: 589-595.

5 Høeg TB, Moldow B, Ellervik C, Klemp K, Erngaard D, LaCour M et al. Danish Rural Eye Study. The association of preschool vision screening with the prevalence of amblyopia. Acta Ophthal; e-pub ahead of print 28 December 2014; doi:10.1111/aos.12639.

6 Marsh-Tootle W, Wall TC, Tootle JS, Person SD, Kristofco RE. Quantitative pediatric vision screening in primary care settings in Alabama. Optom Vis Sci 2008; 85: 849-856.

7 Lang J. A new stereotest. J Pediatr Ophthalmol Strabismus 1983; 20: 72-74.

8 Manny RE, Martinez AT, Fern KD. Testing stereopsis in the preschool child. Is it clinically useful? J Pediatr Ophthalmol Strabismus 1991; 28: 223-231.

9 Ciner EB, Ying G-S, Kulp MT, Maguire MG, Quinn GE, Orel-Bixler D et al. Steroacuity of preschool children with and without vision disorders. Optom Vis Sci 2014; 91: 351-358.

10 Cordonnier M, Dramaix M. Screening for abnormal levels of hyperopia in children: a non-cycloplegic method with a hand-held refractor. Br J Ophthalmol 1998; 82: 1260-1264.

11 Cordonnier M, Dramaix M. Screening for refractive errors in children: accuracy of the hand-held Retinomax to screen for astigmatism. Br J Ophthalmol 1999; 83: 157-161.

12 Vision in Preschoolers (VIP) Study Group. Comparison of preschool vision screening tests as administered by licensed eye care professionals in the VIP study. Ophthalmology 2004; 111: 637-650.

13 Vision in Preschoolers (VIP) Study Group. Impact of confidence number on the screening accuracy of the Retinomax Autorefractor. Optom Vis Sci 2007; 84: 181-188.

14 Ying G, Maguire M, Quinn G, Kulp MT, Cyert LVision in Preschoolers (VIP) Study Group. ROC analysis of the accuracy of noncycloplegic retinoscopy, Retinomax autorefractor, and Sure Sight vision screener for preschool vision screening. Invest Ophthmol Vis Sci 2011; 52: 9658-9664.
15 Cotter SA, Tarczy-Hornoch K, Wang Y, Azen SP, Dilauro A, Borchert $\mathrm{M}$ et al. Visual acuity testability in AfricanAmerican and Hispanic children. Am J Ophthalmol 2007; 144: 663-667.

16 Borchert M, Wang Y, Tarczy-Hornoch K, Cotter S, Deneen J, Azen $S$ et al. Testability of the retinomax autorefractor and IOLMaster in preschool children. Ophthalmology 2008; 115: 1422-1425.

17 Trager MJ, Dirani M, Fan Q. Testability of vision and refraction in preschoolers. The Strabismus, Amblyopia and Refractive Error Study in Singaporean children (STARS). Am J Ophthalmol 2009; 148: 235-241.

18 Pai A-I, Rose KA, Samarawickrama C, Fotedar R, Burlutsky G, Varma $\mathrm{R}$ et al. Testability of refraction, stereopsis and other ocular measures in preschool children. The Sydney Paediatric Eye Disease Study (SPEDS). J AAPOS 2012; 16: 185-192.

19 Slidsborg C, Bangsggard R, Fledelius HC, Jensen H, Greisen G, laCour M. Cerebral damage may be the primary risk factor for visual impairment in preschool children born extremely premature. Arch Ophthalmol 2012; 130: 1410-1417.

20 Slidsborg CC, Olesen HB, Jensen PK, Jensen H, Nissen KR, Greisen $\mathrm{G}$ et al. Treatment for retinopathy of prematurity in Denmark in a ten-year period 1996-2005: Is the incidence increasing. Pediatrics 2008; 121: 97-105.

21 Fledelius HC, Bangsgaard R, Slidsborg S, laCour M. Refraction and visual acuity in a national Danish cohort of 4-year-old children of extremely preterm delivery. Acta Ophthalmol 2015 (in press).

22 Nayak BK, Ghose S, Singh JP. A comparison of cycloplegic and manifest refractions on the NR-1000 F (an objective auto refractometer). Br J Ophthalmol 1987; 71: 73-75.

23 Rosenfield M, Ciuffreda KJ, Hung GK, Gilmartin B. Tonic accommodation: a review, II. Accommodation adaptation and clinical aspects. Ophthalmic Physiol Opt 1994; 14: 265-277.

24 Fledelius HC. Instrument-induced changes in refraction in a pediatric clinical series. Has corneal curvature a role to play? In: Tokoro T (ed). Myopia updates Proceedings of the 6th International Conference on Myopia. Springer: Tokyo, 1998, pp 210-214.

25 Jensen H. Myopia progression in young school children: a prospective study of myopia progression and the effect of a trial with bifocal lenses and beta blocker eye drops. Acta Ophthalmol Suppl 1991; 200: 1-79.

26 Rubin A, Harris WF. Refractive variation during autorefraction: multivariate distribution of refractive status. Optom Vis Sci 1995; 72: 403-410.

27 Fledelius HC. Prematurity and the eye. Ophthalmic 10-year follow-up of children of low and normal birth weight. Acta Ophthalmol Suppl 1976; 128: 3-245. 